Supporting Information

\title{
Comparison of Linear- and Star-Shaped Fused-Ring Electron Acceptors
}

Guilong Cai, ${ }^{\dagger} \ddagger$ Wei Wang, ${ }^{\ddagger}$ Jiadong Zhou,${ }^{\S}$ Yiqun Xiao, ${ }^{\perp}$ Kuan Liu, ${ }^{\ddagger}$ Zengqi Xie, ${ }^{\S}$ Xinhui Lu, ${ }^{\perp}$ Jiarong Lian, ${ }^{\dagger}$ Pengju Zeng, ${ }^{\dagger}$ Yiping Wang, ${ }^{\dagger}$ and Xiaowei Zhan,* ${ }^{\dagger}$

${ }^{\dagger}$ Key Laboratory of Optoelectronic Devices and Systems of Ministry of Education and Guangdong Province, College of Physics and Optoelectronic Engineering, Shenzhen University, Shenzhen 518060, China

$\$$ Department of Materials Science and Engineering, College of Engineering, Key Laboratory of Polymer Chemistry and Physics of Ministry of Education, Peking University, Beijing 100871, China

$\S$ State Key Laboratory of Luminescent Materials and Devices, South China University of Technology, Guangzhou 510640, China

${ }^{\perp}$ Department of Physics, The Chinese University of Hong Kong, New Territories 999077, Hong Kong, China 


\section{Materials}

Unless stated otherwise, all the chemical reagents and solvents used were obtained commercially and were used without further purification. Chloroform (99.9\%), 1,8-diiodooctane (DIO) (97.0\%) and 2-methoxyethanol (99.8\%) were purchased from TCI and J\&K Chemical Inc., respectively; $\mathrm{Zn}\left(\mathrm{CH}_{3} \mathrm{COO}\right)_{2} \cdot 2 \mathrm{H}_{2} \mathrm{O}$ (99.0\%), ethanolamine $(99.5 \%)$ and $\mathrm{MoO}_{3}$ were purchased from Sigma-Aldrich Inc. Toluene and tetrahydrofuran (THF) were distilled from sodium benzophenone under nitrogen. Compound 1 and PM6 $\left(M_{\mathrm{n}}=20.2 \mathrm{~kg} \mathrm{~mol}^{-1}, M_{\mathrm{w}} / M_{\mathrm{n}}=2.0\right)$ were purchased from TCI and 1-Material Inc, respectively. $2 \mathrm{FIC}^{\mathrm{S} 1}$ and $\mathrm{FBD}-\mathrm{CHO}^{\mathrm{S} 2}$ were synthesized according to our reported procedures.

\section{Synthesis of FBTIC and FBDIC}

Compound 2. In a dry three-necked round-bottomed flask, compound 1 (500 mg, $2.0 \mathrm{mmol})$ was dissolved in anhydrous THF $(30 \mathrm{~mL})$. The mixture was deoxygenated with argon for $20 \mathrm{~min}$. At $-78{ }^{\circ} \mathrm{C}$, a solution of LDA (2.0 M in hexane, $5.0 \mathrm{~mL}, 10$ mmol) was added dropwise. After $1 \mathrm{~h}$ of stirring at $-78^{\circ} \mathrm{C}, \mathrm{SnMe}{ }_{3} \mathrm{Cl}(1.0 \mathrm{M}, 7 \mathrm{~mL}, 7$ mmol) was added to this solution. The mixture was warmed up to room temperature and stirred overnight. Ice water $(25 \mathrm{~mL})$ was added and the mixture was extracted with dichloromethane $(3 \times 30 \mathrm{~mL})$. The organic phase was dried over anhydrous $\mathrm{MgSO}_{4}$ and filtered. After removed the solvent under reduced pressure, the residue was recrystallized from ethanol to get the product as a white solid $(1.2 \mathrm{~g}, 80 \%) .{ }^{1} \mathrm{H}$ NMR (400 MHz, $\left.\mathrm{CDCl}_{3}\right): \delta 7.71(\mathrm{~s}, 3 \mathrm{H}), 0.57-0.42(\mathrm{~m}, 27 \mathrm{H})$. MS (MALDI-TOF): m/z $736.8\left(\mathrm{M}^{+}\right)$. Anal. calcd for $\mathrm{C}_{21} \mathrm{H}_{30} \mathrm{~S}_{3} \mathrm{Sn}_{3}$ : C, 34.33; H, 4.12. Found: C, 34.19; H, 
4.16 .

Compound 3. Compound 2 (735 $\mathrm{mg}, \quad 1.0 \quad \mathrm{mmol})$, ethyl 2-bromothiophene-3-carboxylate (1.1 g, $4.5 \mathrm{mmol}), \mathrm{K}_{2} \mathrm{CO}_{3}(2.8 \mathrm{~g}, 20 \mathrm{mmol})$, Aliquat 336 (1 drop), and $\mathrm{Pd}\left(\mathrm{PPh}_{3}\right)_{4}(115 \mathrm{mg}, 0.1 \mathrm{mmol})$ were dissolved in deoxygenated toluene/ $\mathrm{H}_{2} \mathrm{O}(50 \mathrm{~mL}, 2 / 1, \mathrm{v} / \mathrm{v})$. The solution mixture was stirred at $110{ }^{\circ} \mathrm{C}$ for $48 \mathrm{~h}$, and then cooled to room temperature. $100 \mathrm{~mL}$ of water was added and the mixture was extracted with dichloromethane $(2 \times 100 \mathrm{~mL})$. The precipitate was filtered and washed with methanol, $n$-hexane and dichloromethane. The product of compound $\mathbf{3}$ was obtained as a yellow solid (560 mg, 79\%). This compound was directly used for next step reaction without further purification due to limited solubility. MS (MALDI-TOF): $m / z, 707.9\left(\mathrm{M}^{+}\right)$.

FBT. The Grignard reagent was prepared by the following procedure: to a suspension of magnesium turnings (215 $\mathrm{mg}, 9 \mathrm{mmol})$ and 3-4 drops of 1,2-dibromoethane in dry THF (9 $\mathrm{mL})$ was slowly added 1-bromo-4-hexylbenzene (2.15 g, 9 mmol) dropwise, and the mixture was stirred for $2 \mathrm{~h}$. FBT was synthesized by two steps: firstly, to a solution of compound 3 (355 mg, $0.5 \mathrm{mmol})$ in dry THF (10 $\mathrm{mL}$ ) under nitrogen was added the prepared Grignard reagent dropwise at room temperature. The resulting mixture was stirred at reflux for $24 \mathrm{~h} .100 \mathrm{~mL}$ of ammonium chloride saturated solution was added and the mixture was extracted with dichloromethane $(2 \times 100 \mathrm{~mL})$. The combined organic layer was dried over anhydrous $\mathrm{MgSO}_{4}$. After removal of the solvent under reduced pressure, a brown solid was obtained and directly used for next step reaction without further purification. Secondly, 
to a solution of the brown solid in octane $(50 \mathrm{~mL})$ was added acetic acid $(10 \mathrm{~mL})$ and sulfuric acid $(0.05 \mathrm{~mL})$ slowly. The resulting solution was stirred at $70{ }^{\circ} \mathrm{C}$ for $12 \mathrm{~h}$. After removal of octane under reduced pressure, the residue was washed with sodium carbonate saturated aqueous solution $(50 \mathrm{~mL} \times 3)$ and extracted by diethyl ether $(50$ $\mathrm{mL} \times 2$ ). Then, after removal of the solvent under reduced pressure, the residue was purified by column chromatography on silica gel using a mixture solvent as eluent (petroleum ether/dichloromethane, $\mathrm{v} / \mathrm{v}=10 / 1)$ to give a yellow solid (440 $\mathrm{mg}, 2$ steps overall yield 59\%). ${ }^{1} \mathrm{H}$ NMR (400 MHz, $\left.\mathrm{CD}_{2} \mathrm{Cl}_{2}\right): \delta 7.45(\mathrm{~d}, J=6.4 \mathrm{~Hz}, 12 \mathrm{H}), 7.17$ (d, $J=4.7 \mathrm{~Hz}, 3 \mathrm{H}), 7.10(\mathrm{~d}, J=7.5 \mathrm{~Hz}, 12 \mathrm{H}), 7.00(\mathrm{~d}, J=4.9 \mathrm{~Hz}, 3 \mathrm{H}), 2.61-2.56(\mathrm{~m}$, 12H), $1.61-1.54(\mathrm{~m}, 12 \mathrm{H}), 1.33-1.18(\mathrm{~m}, 36 \mathrm{H}), 0.88-0.76(\mathrm{~m}, 18 \mathrm{H}) .{ }^{13} \mathrm{C} \mathrm{NMR}$ $\left(100 \mathrm{MHz}, \mathrm{CD}_{2} \mathrm{Cl}_{2}\right): \delta 163.9,150.8,142.7,139.7,136.1,135.1,132.6,130.5,129.9$, 128.7, 127.6, 123.2, 63.7, 36.1, 32.3, 31.9, 29.6, 23.2, 14.4. MS (MALDI-TOF): $\mathrm{m} / \mathrm{z}$ 1489.5 $\left(\mathrm{M}^{+}\right)$. Anal. calcd for $\mathrm{C}_{99} \mathrm{H}_{108} \mathrm{~S}_{6}: \mathrm{C}, 79.79 ; \mathrm{H}, 7.30$. Found: $\mathrm{C}, 79.85 ; \mathrm{H}, 7.26$.

FBT-CHO. A Vilsmeier reagent was prepared firstly. $4 \mathrm{~mL}$ anhydrous DMF was added to a dry $100 \mathrm{~mL}$ two-necked round bottom flask, and the solution was cooled to $0{ }^{\circ} \mathrm{C}$ and stirred when $0.4 \mathrm{~mL}$ phosphorous oxychloride $\left(\mathrm{POCl}_{3}\right)$ was added by syringe under argon protection. The mixture kept at $0{ }^{\circ} \mathrm{C}$ for $2 \mathrm{~h}$, and then FBT (298 $\mathrm{mg}, 0.2$ mmol) in dry dichloroethane (DCE, $20 \mathrm{~mL}$ ) was added. The mixture solution was allowed to reflux overnight. After cooling to room temperature, $100 \mathrm{~mL}$ water was added to quench the reaction. The mixture was extracted with dichloromethane, and the organic layer was collected, washed with water and dried with anhydrous $\mathrm{MgSO}_{4}$. After removal of the solvent under reduced pressure, the residue was purified by 
column chromatography on silica gel using a mixture solvent as eluent (petroleum ether/dichloromethane, $\mathrm{v} / \mathrm{v}=1 / 1)$ to give a yellow solid $(260 \mathrm{mg}, 83 \%) .{ }^{1} \mathrm{H} \mathrm{NMR}$ $\left(300 \mathrm{MHz}, \mathrm{CDCl}_{3}\right): \delta 9.74(\mathrm{~s}, 3 \mathrm{H}), 7.61(\mathrm{~s}, 3 \mathrm{H}), 7.40(\mathrm{~d}, J=7.9 \mathrm{~Hz}, 12 \mathrm{H}), 7.10(\mathrm{~d}, J=$ $7.9 \mathrm{~Hz}, 12 \mathrm{H}), 2.57(\mathrm{t}, J=7.7 \mathrm{~Hz}, 12 \mathrm{H}), 1.62-1.52(\mathrm{~m}, 12 \mathrm{H}), 1.33-1.18(\mathrm{~m}, 36 \mathrm{H})$, $0.83-0.76(\mathrm{~m}, 18 \mathrm{H}) .{ }^{13} \mathrm{C} \mathrm{NMR}\left(75 \mathrm{MHz}, \mathrm{CDCl}_{3}\right): \delta 182.6,163.5,154.1,145.3,144.7$, $142.7,138.5,135.6,134.0,131.3,129.8,129.3,128.6,63.6,35.6,31.8,31.2,29.1$, 22.7, 14.2. MS (MALDI-TOF): $m / z 1573.5\left(\mathrm{M}^{+}\right)$. Anal. calcd for $\mathrm{C}_{102} \mathrm{H}_{108} \mathrm{O}_{3} \mathrm{~S}_{6}: \mathrm{C}$, 77.82; H, 6.91. Found: C, 77.84; H, 6.95.

FBTIC. To a three-necked round bottom flask were added FBT-CHO (157 mg, $0.1 \mathrm{mmol}), 2$ FIC (92 mg, $0.4 \mathrm{mmol})$, pyridine (0.15 mL) and chloroform $(20 \mathrm{~mL})$. The mixture was deoxygenated with nitrogen for $20 \mathrm{~min}$ and then stirred at reflux for $12 \mathrm{~h}$. After cooling to room temperature, the mixture was poured into methanol (200 $\mathrm{mL}$ ) and filtered. The residue was purified by column chromatography on silica gel using a mixture solvent as eluent (petroleum ether/dichloromethane, $\mathrm{v} / \mathrm{v}=1 / 1$ ) to give a blue solid (175 mg, 80\%). ${ }^{1} \mathrm{H}$ NMR $\left(500 \mathrm{MHz}, \mathrm{CDCl}_{3}\right): \delta 8.79(\mathrm{~s}, 3 \mathrm{H}), 8.53(\mathrm{dd}, J=$ 9.9, 6.3 Hz, 3H), $7.66(\mathrm{t}, J=7.4 \mathrm{~Hz}, 3 \mathrm{H}), 7.59(\mathrm{~s}, 3 \mathrm{H}), 7.39(\mathrm{~d}, J=8.0 \mathrm{~Hz}, 12 \mathrm{H}), 7.14$ $(\mathrm{d}, J=8.0 \mathrm{~Hz}, 12 \mathrm{H}), 2.63-2.53(\mathrm{~m}, 12 \mathrm{H}), 1.69-1.56(\mathrm{~m}, 12 \mathrm{H}), 1.34-1.20(\mathrm{~m}$, 36H), $0.88-0.71(\mathrm{~m}, 18 \mathrm{H}) .{ }^{13} \mathrm{C}$ NMR $\left(125 \mathrm{MHz}, \mathrm{CDCl}_{3}\right): \delta$ 186.4, 164.6, 158.3, $156.8,155.7,155.6,154.5,153.7,153.5,143.2,140.3,139.2,138.8,138.5,138.4$, $136.7,136.7,134.6,134.5,133.2,129.7,129.3,128.8,120.7,115.3,115.1,114.4$, 112.8, 112.6, 69.5, 63.6, 35.7, 31.8, 31.2, 29.2, 22.7, 14.2. MS (MALDI-TOF): $\mathrm{m} / \mathrm{z}$ $2210.6\left(\mathrm{M}^{+}\right)$. Anal. calcd for $\mathrm{C}_{138} \mathrm{H}_{114} \mathrm{~F}_{6} \mathrm{~N}_{6} \mathrm{O}_{3} \mathrm{~S}_{6}: \mathrm{C}, 74.97 ; \mathrm{H}, 5.20 ; \mathrm{N}, 3.80$. Found: $\mathrm{C}$, 
74.99; H, 5.23; N, 3.78 .

FBDIC. To a three-necked round bottom flask were added FBD-CHO (108 mg, $0.1 \mathrm{mmol}), 2 \mathrm{FIC}(70 \mathrm{mg}, 0.3 \mathrm{mmol})$, pyridine $(0.15 \mathrm{~mL})$ and chloroform $(20 \mathrm{~mL})$. The mixture was deoxygenated with nitrogen for $20 \mathrm{~min}$ and then stirred at reflux for $12 \mathrm{~h}$. After cooling to room temperature, the mixture was poured into methanol (200 $\mathrm{mL}$ ) and filtered. The residue was purified by column chromatography on silica gel using a mixture solvent as eluent (petroleum ether/dichloromethane, $\mathrm{v} / \mathrm{v}=1 / 1$ ) to give a blue-green solid (122 mg, 84\%). ${ }^{1} \mathrm{H}$ NMR (400 MHz, $\left.\mathrm{CDCl}_{3}\right): \delta 8.86(\mathrm{~s}, 2 \mathrm{H}), 8.53$ (dd, $J=9.9,6.4 \mathrm{~Hz}, 2 \mathrm{H}), 8.02(\mathrm{~s}, 2 \mathrm{H}), 7.72(\mathrm{~s}, 2 \mathrm{H}), 7.67(\mathrm{t}, J=7.5 \mathrm{~Hz}, 2 \mathrm{H}), 7.16(\mathrm{~d}, J$ $=8.4 \mathrm{~Hz}, 8 \mathrm{H}), 7.10(\mathrm{~d}, J=8.4 \mathrm{~Hz}, 8 \mathrm{H}), 2.63-2.49(\mathrm{~m}, 8 \mathrm{H}), 1.61-1.54(\mathrm{~m}, 8 \mathrm{H})$, $1.33-1.21(\mathrm{~m}, 24 \mathrm{H}), 0.90-0.81(\mathrm{~m}, 12 \mathrm{H}) .{ }^{13} \mathrm{C} \mathrm{NMR}\left(100 \mathrm{MHz}, \mathrm{CDCl}_{3}\right): \delta 186.3$, $163.2,158.3,156.7,156.5,144.3,142.9,140.6,139.0,138.8,138.6,137.2,131.9$, $129.1,128.1,120.9,118.4,115.3,115.1,114.5,114.4,112.8,112.7,69.4,62.9,35.7$, 31.8, 31.4, 29.2, 22.7, 14.2. MS (MALDI-TOF): $m / z 1499.7\left(\mathrm{M}^{+}\right)$. Anal. calcd for $\mathrm{C}_{94} \mathrm{H}_{78} \mathrm{~F}_{4} \mathrm{~N}_{4} \mathrm{O}_{2} \mathrm{~S}_{4}: \mathrm{C}, 75.27 ; \mathrm{H}, 5.24 ; \mathrm{N}, 3.74$. Found: C, 75.33; H, 5.25; N, 3.72.

\section{Characterization}

The ${ }^{1} \mathrm{H}$ NMR and ${ }^{13} \mathrm{C}$ NMR spectra were measured by Bruker AVANCE 300, 400 or $500 \mathrm{MHz}$ spectrometer. Mass spectra were performed by Bruker Daltonics Biflex III MALDI-TOF Analyzer in the MALDI mode. Elemental analyses were measured through a Flash EA 1112 elemental analyzer. Thermogravimetric analysis (TGA) measurements were carried out using a Shimadzu thermogravimetric analyzer (Model DTG-60) under flowing nitrogen gas at a heating rate of $10{ }^{\circ} \mathrm{C} \mathrm{min}^{-1}$. The 
absorption spectra were measured using the JASCO-570 spectrophotometer (JASCO. Inc., Japan) in solution (chloroform) and the thin film (on a quartz substrate). Electrochemical measurements were carried out under nitrogen in a solution of tetra- $n$-butylammonium hexafluorophosphate $\left(\left[{ }^{\mathrm{n}} \mathrm{Bu}_{4} \mathrm{~N}\right]^{+}\left[\mathrm{PF}_{6}\right]^{-}\right)(0.1 \mathrm{M})$ in $\mathrm{CH}_{3} \mathrm{CN}$ employing a computer-controlled CHI660C electrochemical workstation, a glassy carbon working electrode coated with FBTIC or FBDIC film, a $\mathrm{Ag} / \mathrm{AgCl}$ reference electrode, and a platinum-wire auxiliary electrode. The potentials were referenced to a ferrocenium/ferrocene $\left(\mathrm{FeCp}_{2}{ }^{+/ 0}\right)$ couple using ferrocene as an external standard. Atomic force microscope (AFM) images were measured on Multimode 8 scanning probe microscopy (Bruker Daltonics, United States) in the tapping mode.

\section{Suitable crystal XRD measurements}

Suitable crystals were selected and recorded on Rigaku XtaLAB P2000 FR-X with a rotating copper anode and a Pilatus $200 \mathrm{~K}$ detector. The crystal was kept at 150 $\mathrm{K}$ during data collection. The structure was solved with the ShelXT program using intrinsic phasing and refined with the ShelXL refinement package using least squares minimization. CCDC numbers 1911552 and 1911553 contain the supplementary crystallographic data for this paper, which can be obtained free of charge from The Cambridge Crystallographic Data Centre via www.ccdc.cam.ac.uk/data_request/cif.

\section{GIWAXS and GISAXS measurements}

GIWAXS measurements were accomplished with a Xeuss 2.0 SAXS/WAXS laboratory beamline using a Cu X-ray source (8.05 keV, $1.54 \AA$ A) and a Pilatus3R $300 \mathrm{~K}$ detector. The incidence angle is $0.2^{\circ}$. GISAXS measurement was conducted at 
19U2 SAXS beamline at Shanghai Synchrotron Radiation Facility, Shanghai, China, also using the $0.15^{\circ}$ incident angle with $10 \mathrm{keV}$ primary beam. Both GIW AXS and GISAXS samples are prepared on silicon substrate by spin coating.

\section{Device fabrication and characterization}

All the devices are based on an inverted sandwich structure, patterned indium-tin oxide glass (ITO glass)/zinc oxide ( $\mathrm{ZnO})\left(c a .30\right.$ nm)/PM6: acceptor/MoO $\mathrm{MoO}_{3}(\mathrm{ca} .5$ $\mathrm{nm}) / \mathrm{Ag}(\mathrm{ca} .80 \mathrm{~nm})$. First, ITO glass (sheet resistance $=15 \Omega \cdot \square^{-1}$ ) was continuously pre-cleaned in the ultrasonic bath with de-ionized water, acetone and isopropanol. Then, a $\mathrm{ZnO}$ layer was spin-coated at $4000 \mathrm{r} \cdot \mathrm{min}^{-1}$ onto the ITO glass from $\mathrm{ZnO}$ precursor solution (prepared by dissolving $0.1 \mathrm{~g}$ of zinc acetate dihydrate $\left(\mathrm{Zn}\left(\mathrm{CH}_{3} \mathrm{COO}\right)_{2} \cdot 2 \mathrm{H}_{2} \mathrm{O}\right)$ and $0.03 \mathrm{~mL}$ of ethanolamine $\left(\mathrm{NH}_{2} \mathrm{CH}_{2} \mathrm{CH}_{2} \mathrm{OH}\right)$ in $1 \mathrm{~mL}$ of 2-methoxyethanol $\left(\mathrm{CH}_{3} \mathrm{OCH}_{2} \mathrm{CH}_{2} \mathrm{OH}\right)$ ), followed by baking at $200{ }^{\circ} \mathrm{C}$ for $30 \mathrm{~min}$. The active layer blend $\left(10.0 \mathrm{mg} \mathrm{mL} L^{-1}\right.$ in chloroform) was spin-coated at $2000 \mathrm{r} \cdot \mathrm{min}^{-1}$ onto the $\mathrm{ZnO}$ layer to form the photoactive layer. $\mathrm{A} \mathrm{MoO}_{3}$ layer and $\mathrm{Ag}$ layer were then evaporated under vacuum $\left(c a \cdot 10^{-5} \mathrm{~Pa}\right)$ to form the anode electrode. The $J-V$ curves were measured using a computer-controlled B2912A Precision Source/Measure Unit (Agilent Technologies, United States). An XES-70S1 (SAN-EI Electric Co., Ltd., Japan) solar simulator (AAA grade, $70 \mathrm{~mm} \times 70 \mathrm{~mm}$ ) coupled with AM 1.5G solar spectrum filters was used as the light source, and the optical power at the sample was $100 \mathrm{~mW} \mathrm{~cm}^{-2}$. The measured area of the active device was $4 \mathrm{~mm}^{2}$. A 2 $\mathrm{cm} \times 2 \mathrm{~cm}$ monocrystalline silicon reference cell (SRC-1000-TC-QZ) was purchased from VLSI Standards Inc. The external quantum efficiency (EQE) spectra were 
measured using a Solar Cell Spectral Response Measurement System QE-R3011 (Enlitech Co., Ltd.). The light intensity at each wavelength was calibrated through standard single crystal Si photovoltaic cell.

\section{SCLC measurements}

Hole-only or electron-only devices were fabricated as follows, ITO/PEDOT:PSS/active layer/Au for holes and ITO/ZnO/active layer/Ca/Al for electrons. The mobility was extracted by fitting the $J-V$ curves using space charge limited current (SCLC) method, ${ }^{\mathrm{S3}}$ which follows $J=$ $(9 / 8) \mu \varepsilon_{\mathrm{r}} \varepsilon_{0} V^{2} \exp \left(0.89\left(V / E_{0} L\right)^{0.5}\right) / L^{3}$.

Here, $J$ refers to the current density, $\mu$ is hole or electron mobility, $\varepsilon_{r}$ is relative dielectric constant of the transport medium, which is equal to $3, \varepsilon_{0}$ is the permittivity of free space $\left(8.85 \times 10^{-12} \mathrm{~F} \mathrm{~m}^{-1}\right), V=V_{\text {appl }}-V_{\mathrm{bi}}$, where $V_{\mathrm{appl}}$ is the applied voltage to the device, and $V_{\mathrm{bi}}$ is the built-in voltage due to the difference in work function of the two electrodes (for hole-only diodes, $V_{\mathrm{bi}}$ is $0.2 \mathrm{~V}$; for electron-only diodes, $V_{\mathrm{bi}}$ is $0 \mathrm{~V}$ ). $E_{0}$ is characteristic field, $L$ is the thickness of the active layer and was measured by Dektak XT (Bruker). 
(a)

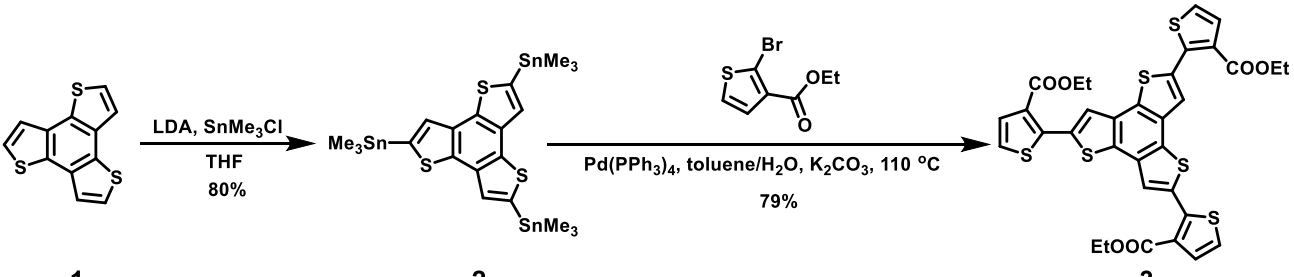

1

2

3

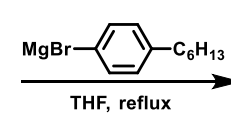

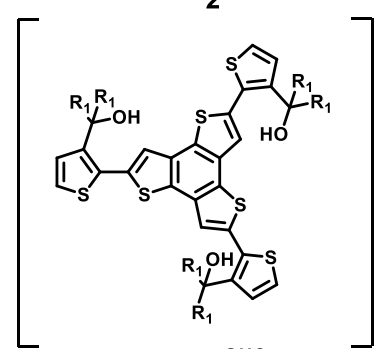

octane $/ \mathrm{AcOH} / \mathrm{H}_{2} \mathrm{SO}_{4}, 70{ }^{\circ} \mathrm{C}$ 2 steps overall yield $59 \%$

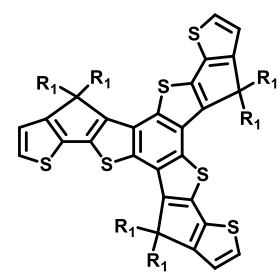

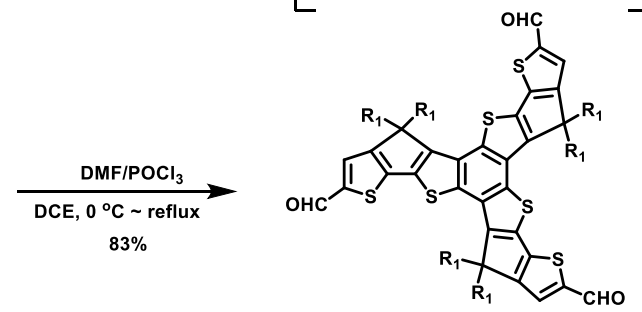

FBT

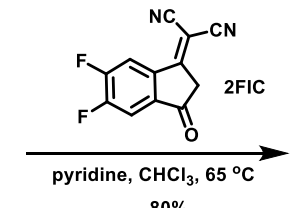

$80 \%$

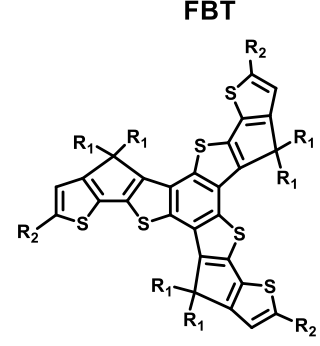

FBT-CHO

FBTIC

(b)

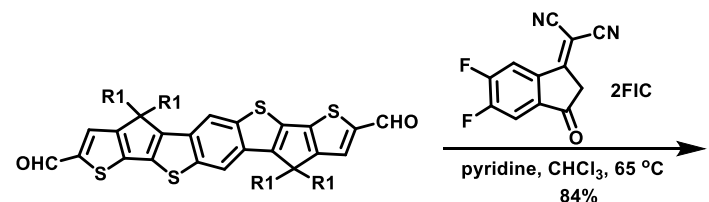

FBD-CHO

$$
\mathrm{R}_{1}=-\cdots-\mathrm{C}_{6} \mathrm{H}_{13}
$$

Scheme S1. Synthetic routes to FBTIC and FBDIC.

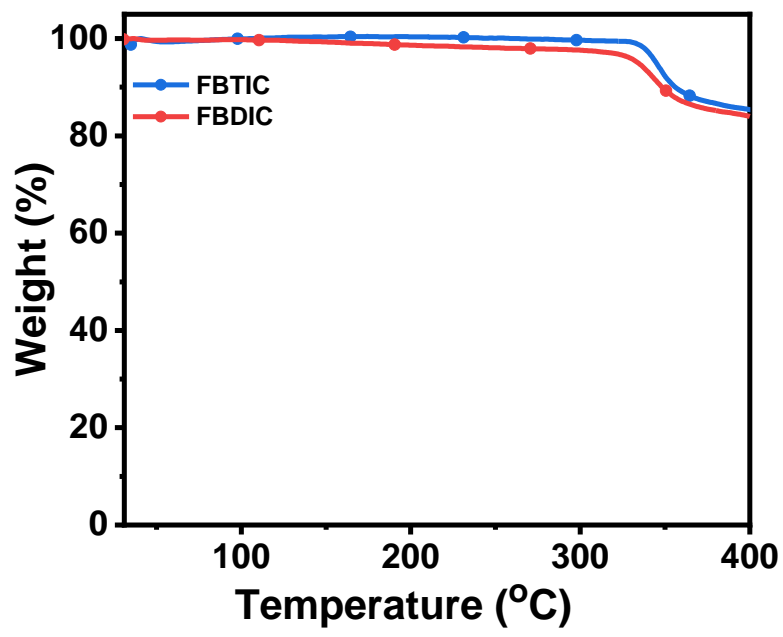

Figure S1. TGA curves of FBTIC and FBDIC. 


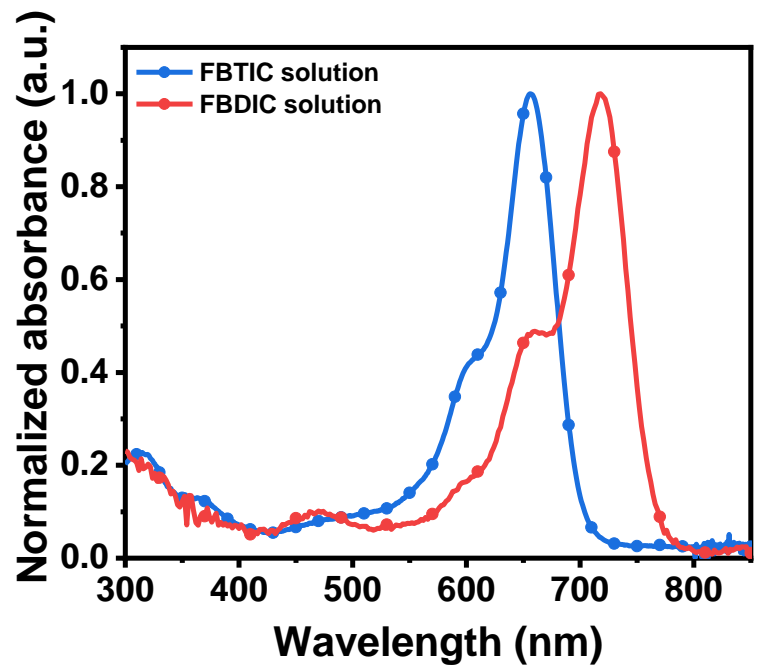

Figure S2. FBTIC and FBDIC absorption spectra in chloroform solution.

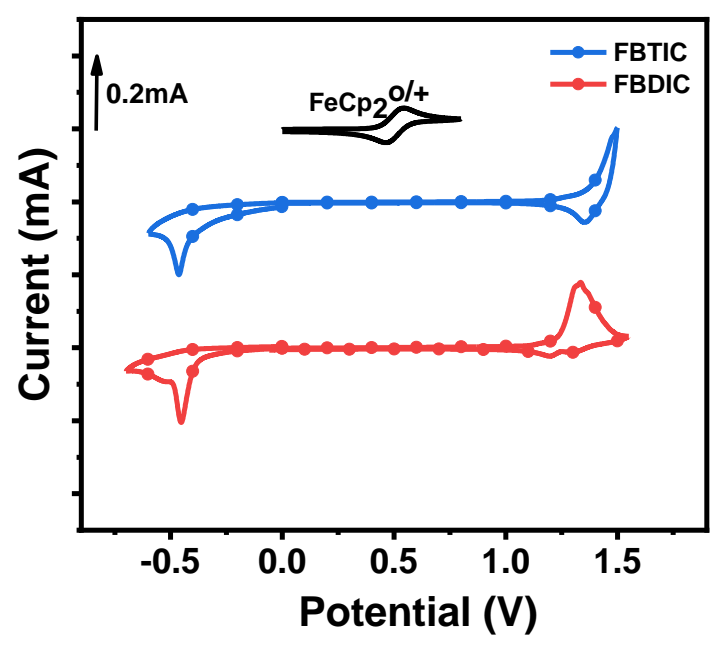

Figure S3. Cyclic voltammograms for FBTIC and FBDIC in $\mathrm{CH}_{3} \mathrm{CN} / 0.1 \mathrm{M} \mathrm{Bu}_{4} \mathrm{NPF}_{6}$ at $100 \mathrm{mV} \mathrm{s}^{-1}$; the horizontal scale refers to an $\mathrm{Ag} / \mathrm{AgCl}$ electrode as a reference electrode. 


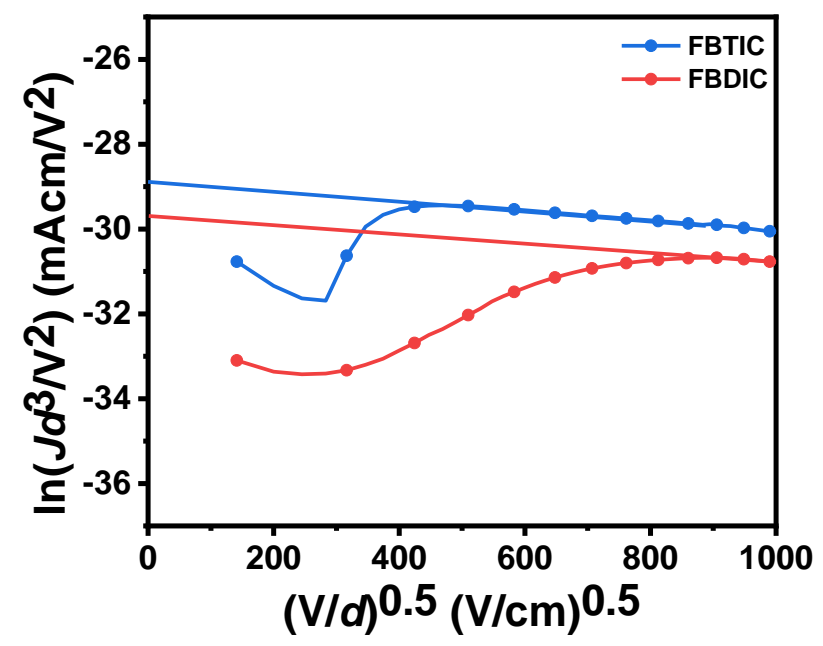

Figure S4. $J$ - $V$ characteristics in the dark for electron-only devices based on FBTIC and FBDIC.
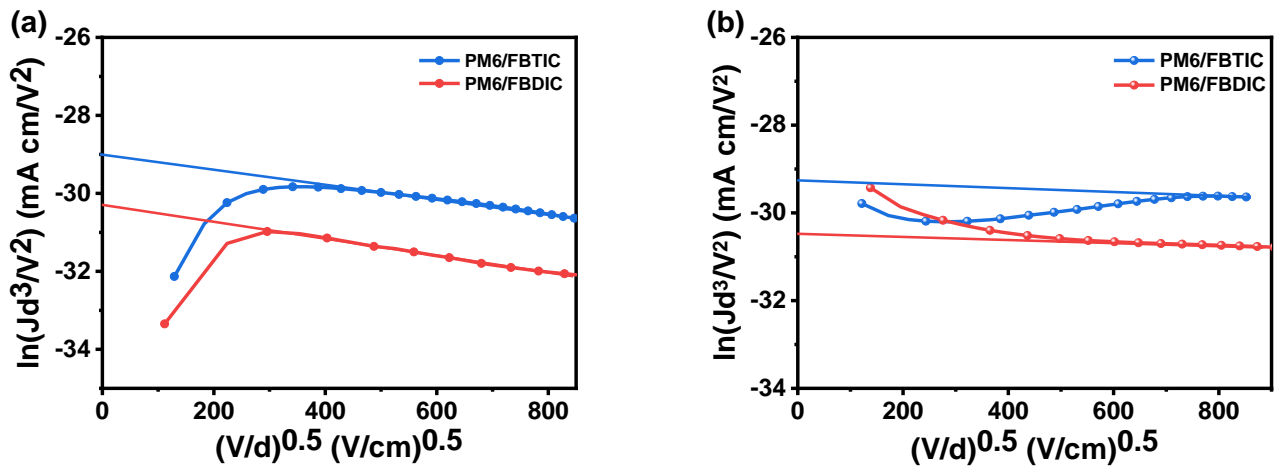

Figure S5. $J-V$ characteristics in the dark for (a) hole-only and (b) electron-only devices based on PM6/acceptor blends. 


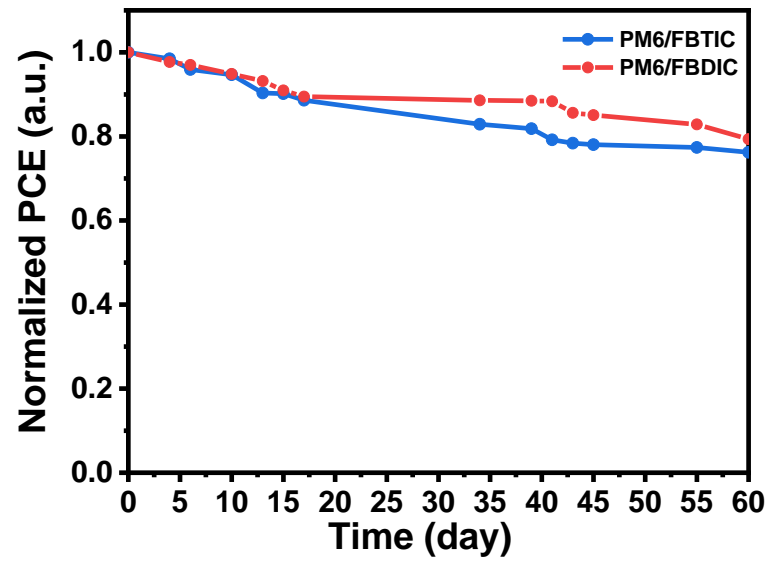

Figure S6. The air stability of OSCs without encapsulation based on PM6/acceptor blends.
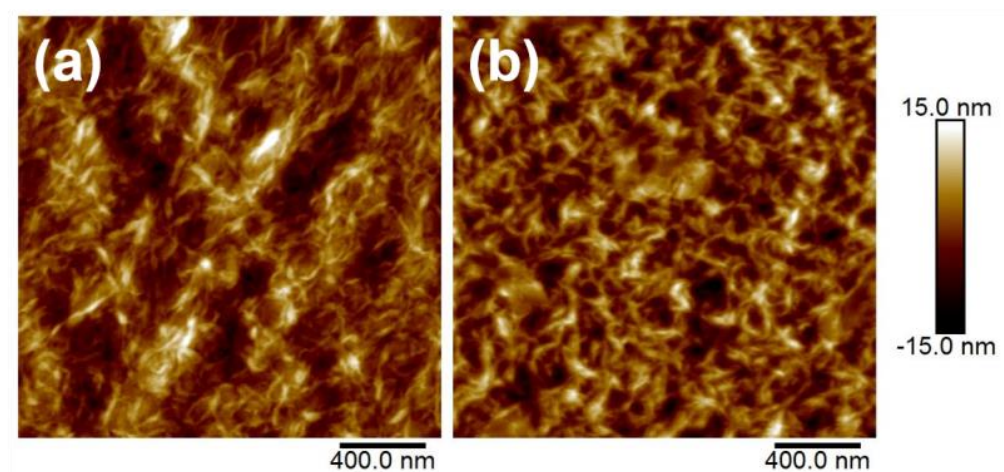

Figure S7. AFM height images of (a) PM6/FBTIC and (b) PM6/FBDIC blends under optimized device conditions. 
(a)

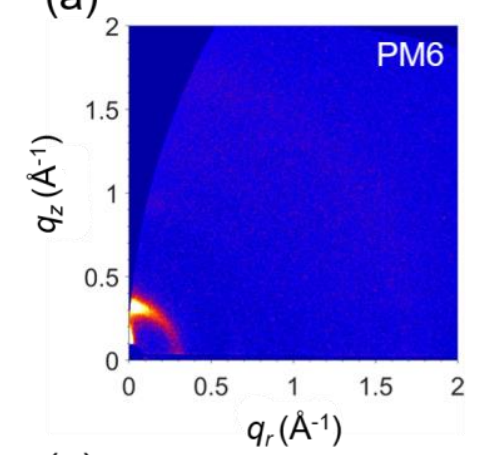

(c)

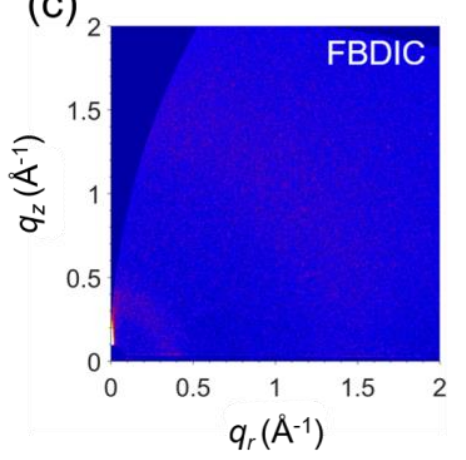

(b)

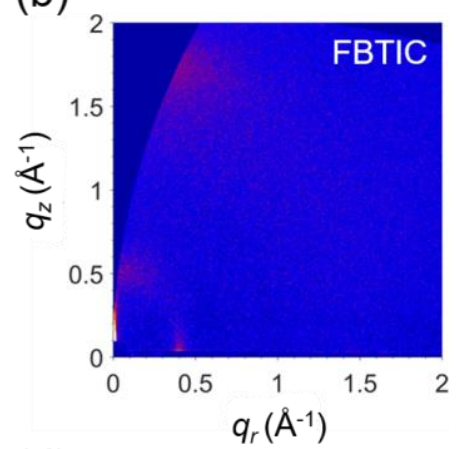

(d)

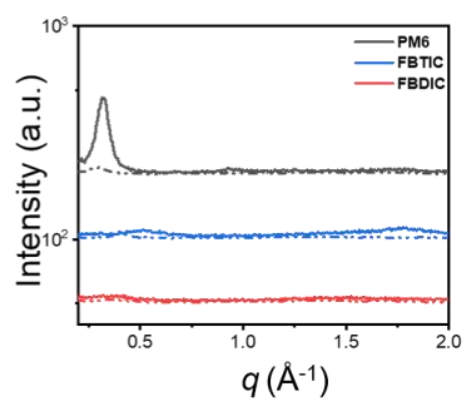

Figure S8. 2D GIWAXS patterns of (a) PM6, (b) FBTIC and (c) FBDIC pure films; (d) the corresponding intensity profiles along the in-plane (dashed line) and out-of-plane (solid line) directions. 
Table S1. Crystal data and structure refinement for FBTIC and FBDIC.

\begin{tabular}{|c|c|c|}
\hline Identification code & FBTIC & FBDIC \\
\hline Empirical formula & $\mathrm{C}_{138} \mathrm{H}_{114} \mathrm{~F}_{6} \mathrm{~N}_{6} \mathrm{O}_{3} \mathrm{~S}_{6}$ & $\mathrm{C}_{94} \mathrm{H}_{78} \mathrm{~F}_{4} \mathrm{~N}_{4} \mathrm{O}_{2} \mathrm{~S}_{4}$ \\
\hline Formula weight & 2210.71 & 1499.84 \\
\hline Temperature/K & $149.99(10)$ & $149.97(10)$ \\
\hline Crystal system & triclinic & monoclinic \\
\hline Space group & P-1 & $\mathrm{P} 2{ }_{1} / \mathrm{c}$ \\
\hline $\mathrm{a} / \AA$ & $14.9531(4)$ & $34.4434(18)$ \\
\hline $\mathrm{b} / \AA$ & $18.4275(7)$ & $11.1624(3)$ \\
\hline $\mathrm{c} / \AA ̊$ & $24.5510(7)$ & $19.7605(9)$ \\
\hline$\alpha /{ }^{\circ}$ & $102.982(3)$ & 90 \\
\hline$\beta /{ }^{\circ}$ & $99.472(2)$ & $97.631(5)$ \\
\hline$\gamma /{ }^{\circ}$ & $101.245(3)$ & 90 \\
\hline Volume $/ \AA^{3}$ & $6310.0(4)$ & $7530.1(6)$ \\
\hline $\mathrm{Z}$ & 2 & 4 \\
\hline$\rho_{\text {calc }} \mathrm{g} / \mathrm{cm}^{3}$ & 1.164 & 1.323 \\
\hline$\mu / \mathrm{mm}^{-1}$ & 1.497 & 1.683 \\
\hline $\mathrm{F}(000)$ & 2316 & 3144 \\
\hline Crystal size $/ \mathrm{mm}^{3}$ & $0.08 \times 0.06 \times 0.04$ & $0.1 \times 0.06 \times 0.04$ \\
\hline Radiation & $\mathrm{CuK} \alpha(\lambda=1.54184)$ & $\mathrm{CuK} \alpha(\lambda=1.54184)$ \\
\hline $2 \Theta$ range for data collection ${ }^{\circ}$ & 5.072 to 134.158 & 5.178 to 147.836 \\
\hline Index ranges & $\begin{array}{l}-17 \leq \mathrm{h} \leq 16 \\
-11 \leq \mathrm{k} \leq 21 \\
-29 \leq 1 \leq 29\end{array}$ & $\begin{array}{l}-39 \leq \mathrm{h} \leq 42 \\
-9 \leq \mathrm{k} \leq 13 \\
-23 \leq 1 \leq 24\end{array}$ \\
\hline Reflections collected & 66277 & 36243 \\
\hline Independent reflections & $\begin{array}{l}22107\left[R_{\text {int }}=0.0649,\right. \\
\left.R_{\text {sigma }}=0.0643\right]\end{array}$ & $\begin{array}{l}14746\left[\mathrm{R}_{\text {int }}=0.0846,\right. \\
\left.\mathrm{R}_{\text {sigma }}=0.1137\right]\end{array}$ \\
\hline Data/restraints/parameters & $22107 / 200 / 1438$ & $14746 / 3 / 977$ \\
\hline Goodness-of-fit on $\mathrm{F}^{2}$ & 1.191 & 1.033 \\
\hline Final R indexes $[\mathrm{I}>=2 \sigma(\mathrm{I})]$ & $\begin{array}{l}\mathrm{R}_{1}=0.1202 \\
\mathrm{wR}_{2}=0.3255\end{array}$ & $\begin{array}{l}\mathrm{R}_{1}=0.1040 \\
\mathrm{wR}_{2}=0.2601\end{array}$ \\
\hline Final $\mathrm{R}$ indexes [all data] & $\begin{array}{l}\mathrm{R}_{1}=0.1800 \\
\mathrm{wR}_{2}=0.3766\end{array}$ & $\begin{array}{l}\mathrm{R}_{1}=0.2025 \\
\mathrm{wR}_{2}=0.3219\end{array}$ \\
\hline Largest diff. peak/hole / e $\AA^{-3}$ & $0.62 /-0.60$ & $1.10 /-0.33$ \\
\hline
\end{tabular}


Table S2. Device data of as-cast OSCs based on PM6/acceptor with different D/A ratio.

\begin{tabular}{cccccc}
\hline acceptor & $\begin{array}{c}\mathrm{D} / \mathrm{A} \\
(\mathrm{w} / \mathrm{w})\end{array}$ & $\begin{array}{c}V_{\mathrm{OC}}{ }^{a} \\
(\mathrm{~V})\end{array}$ & $\begin{array}{c}J_{\mathrm{SC}}{ }^{a} \\
\left(\mathrm{~mA} \mathrm{~cm}{ }^{-2}\right)\end{array}$ & $\begin{array}{c}\mathrm{FF}^{a} \\
(\%)\end{array}$ & $\begin{array}{c}\mathrm{PCE}^{a} \\
(\%)\end{array}$ \\
\hline & $1.2 / 1$ & 0.976 & 13.1 & 70.6 & 9.04 \\
& & $(0.969 \pm 0.004)$ & $(13.2 \pm 0.1)$ & $(69.7 \pm 1.0)$ & $(8.89 \pm 0.13)$ \\
FBTIC & $1 / 1$ & 0.962 & 14.3 & 72.3 & 9.94 \\
& & $(0.955 \pm 0.004)$ & $(14.3 \pm 0.2)$ & $(70.5 \pm 1.0)$ & $(9.62 \pm 0.19)$ \\
& $1 / 1.2$ & 0.921 & 14.4 & 72.5 & 9.60 \\
& $1.2 / 1$ & 0.877 & 18.3 & 65.7 & 10.5 \\
& & $(0.871 \pm 0.006)$ & $(17.7 \pm 0.3)$ & $(64.6 \pm 1.1)$ & $(9.94 \pm 0.29)$ \\
& $1 / 1$ & 0.862 & 18.9 & 68.3 & 11.1 \\
FBDIC & & $(0.859 \pm 0.008)$ & $(18.4 \pm 0.4)$ & $(66.7 \pm 1.5)$ & $(10.5 \pm 0.3)$ \\
& $1 / 1.2$ & 0.884 & 17.8 & 62.5 & 9.85 \\
& & $(0.880 \pm 0.005)$ & $(17.7 \pm 0.2)$ & $(60.4 \pm 1.7)$ & $(9.44 \pm 0.38)$ \\
\hline
\end{tabular}

${ }^{a}$ Average values (in parenthesis) are obtained from 20 devices. 
Table S3. Device data of the OSCs based on PM6/acceptor (D/A=1/1) with different DIO content in chloroform.

\begin{tabular}{cccccc}
\hline acceptor & $\begin{array}{c}\mathrm{DIO} \\
(\%)\end{array}$ & $\begin{array}{c}V_{\mathrm{OC}}{ }^{a} \\
(\mathrm{~V})\end{array}$ & $\begin{array}{c}J_{\mathrm{SC}}{ }^{a} \\
\left(\mathrm{~mA} \mathrm{~cm}^{-2}\right)\end{array}$ & $\begin{array}{c}\mathrm{FF}^{a} \\
(\%)\end{array}$ & $\begin{array}{c}\mathrm{PCE}^{a} \\
(\%)\end{array}$ \\
\hline \multirow{2}{*}{ FBTIC } & 0.2 & 0.947 & 14.1 & 75.4 & 10.1 \\
& & $(0.937 \pm 0.005)$ & $(14.4 \pm 0.2)$ & $(72.6 \pm 1.4)$ & $(9.66 \pm 0.22)$ \\
\hline \multirow{2}{*}{ FBDIC } & 0.1 & 0.849 & 19.4 & 69.6 & 11.5 \\
& & $(0.850 \pm 0.003)$ & $(19.3 \pm 0.2)$ & $(68.3 \pm 1.7)$ & $(11.2 \pm 0.3)$ \\
& & 0.846 & 20.6 & 70.7 & 12.3 \\
& 0.3 & $(0.850 \pm 0.006)$ & $(19.6 \pm 0.6)$ & $(71.0 \pm 0.9)$ & $(11.8 \pm 0.3)$ \\
& & $(0.841 \pm 0.002)$ & $(18.6 \pm 0.3)$ & $(69.0 \pm 0.8)$ & $(10.8 \pm 0.2)$ \\
\hline
\end{tabular}

${ }^{a}$ Average values (in parenthesis) are obtained from 20 devices.

Table S4. Charge mobilities of pure and blend films measured by SCLC method.

\begin{tabular}{cccc}
\hline active layer & $\mu_{\mathrm{h}}\left(\mathrm{cm}^{2} \mathrm{~V}^{-1} \mathrm{~s}^{-1}\right)$ & $\mu_{\mathrm{e}}\left(\mathrm{cm}^{2} \mathrm{~V}^{-1} \mathrm{~s}^{-1}\right)$ & $\mu_{\mathrm{h}} / \mu_{\mathrm{e}}$ \\
\hline FBTIC & - & $1.7 \times 10^{-3}$ & - \\
FBDIC & - & $7.5 \times 10^{-4}$ & - \\
PM6/FBTIC & $1.5 \times 10^{-3}$ & $1.2 \times 10^{-3}$ & 1.3 \\
PM6/FBDIC & $5.5 \times 10^{-4}$ & $3.3 \times 10^{-4}$ & 1.7 \\
\hline
\end{tabular}




\section{References:}

(S1) Dai, S.; Zhao, F.; Zhang, Q.; Lau, T. K.; Li, T.; Liu, K.; Ling, Q.; Wang, C.; Lu, X.; You, W.; Zhan, X. Fused Nonacyclic Electron Acceptors for Efficient Polymer Solar Cells. J. Am. Chem. Soc. 2017, 139, 1336-1343.

(S2) Wang, J.; Wang, W.; Wang, X.; Wu, Y.; Zhang, Q.; Yan, C.; Ma, W.; You, W.;

Zhan, X. Enhancing Performance of Nonfullerene Acceptors via Side-Chain Conjugation Strategy. Adv. Mater. 2017, 29, 1702125.

(S3) Malliaras, G. G.; Salem, J. R.; Brock, P. J.; Scott, C. Electrical Characteristics and Efficiency of Single-Layer Organic Light-Emitting Diodes. Phys. Rev. B 1998, $58,13411-13414$. 